\title{
Large-Area Wireless Sensor System for Ambient Assisted Living
}

\author{
A. Steinhage, C. Lauterbach, A. Techmer \\ Future-Shape GmbH, Höhenkirchen-Siegertsbrunn, Germany \\ axel.steinhage@future-shape.com
}

\begin{abstract}
:
We present a large-area wireless sensor system underneath the floor covering which is based on smart textiles. Typically 32 sensors per square meter invisibly installed underneath soft or hard floor coverings measure the change of capacitance induced by people walking across the floor such that the location and the state of persons can be detected. The system supports applications in many domains such as security, energy saving, comfort, entertainment, marketing and robotics. Within this paper we present examples from Ambient Assisted Living (AAL) where the floor supports carers and allows the elderly to remain independent as long as possible.
\end{abstract}

Key words: capacitive sensors, wireless network, smart textiles, location detector, home automation

\section{Introduction}

Being able to detect the location and state of people indoor allows for multiple applications in many domains of daily life. Some of these applications, such as access control systems for example, require to capture not only the position but also the identity of a person. This can be achieved, for instance, by means of wearable radio tags which can be located by exploiting the change of the electromagnetic field geometry induced by the moving tags. However, in many applications an identification is not necessary or even not allowed. This is the case, for instance, when only the number of people in public spaces is of interest or when dangerous situations should lead to an alarm independent of who caused the situation. Within this paper we present applications from the domain of Ambient Assisted Living (AAL) [1,2], the aim of which is to assist elderly people in their home or in institutional care e.g. by automatically detecting a fall or immobility. Specifically in this area, a sensor system is required which works reliably even when the person forgets to carry or is unable to use any wearable alarm button. Furthermore, for the person in need of assistance, it is often mandatory that their privacy is protected and that sensors are not obvious to visitors in order to prevent the feeling of being stigmatized as not being independent anymore. For the latter reasons also camera-based systems seem not to be the best solution.
Within this paper we present a technology which complies with the aforementioned requirements in an ideal way: by means of a large-area sensor underlay which is invisibly installed underneath the floor covering, the location of people can be detected with high spatial and temporal resolution. Based on intelligent sensor data processing not only steps, direction and velocity of persons can be analyzed, but it is also possible to detect falls or immobility.

In the following, we describe the technology, the measurement principle and the data analysis before we show a concrete implementation and different variants of the system.

\section{System Description}

The SensFloor ${ }^{\circledR}$ system integrates a grid of capacitive proximity sensor areas and microelectronic modules into a textile composite underlay. This large-area sensor system is compatible with virtually any type of flooring except for conductive materials.

A schematic of the system is shown in Fig. 1. When a person walks across the floor, sensor events are detected by the triangular sensor areas beneath the feet. The sensor areas are obtained by laminating a thin layer of conductive fleece onto a $2 \mathrm{~mm}$ thick nonconductive conventional sound insulating fleece and structuring the conductive fleece into sensor areas and power supply lanes for the 


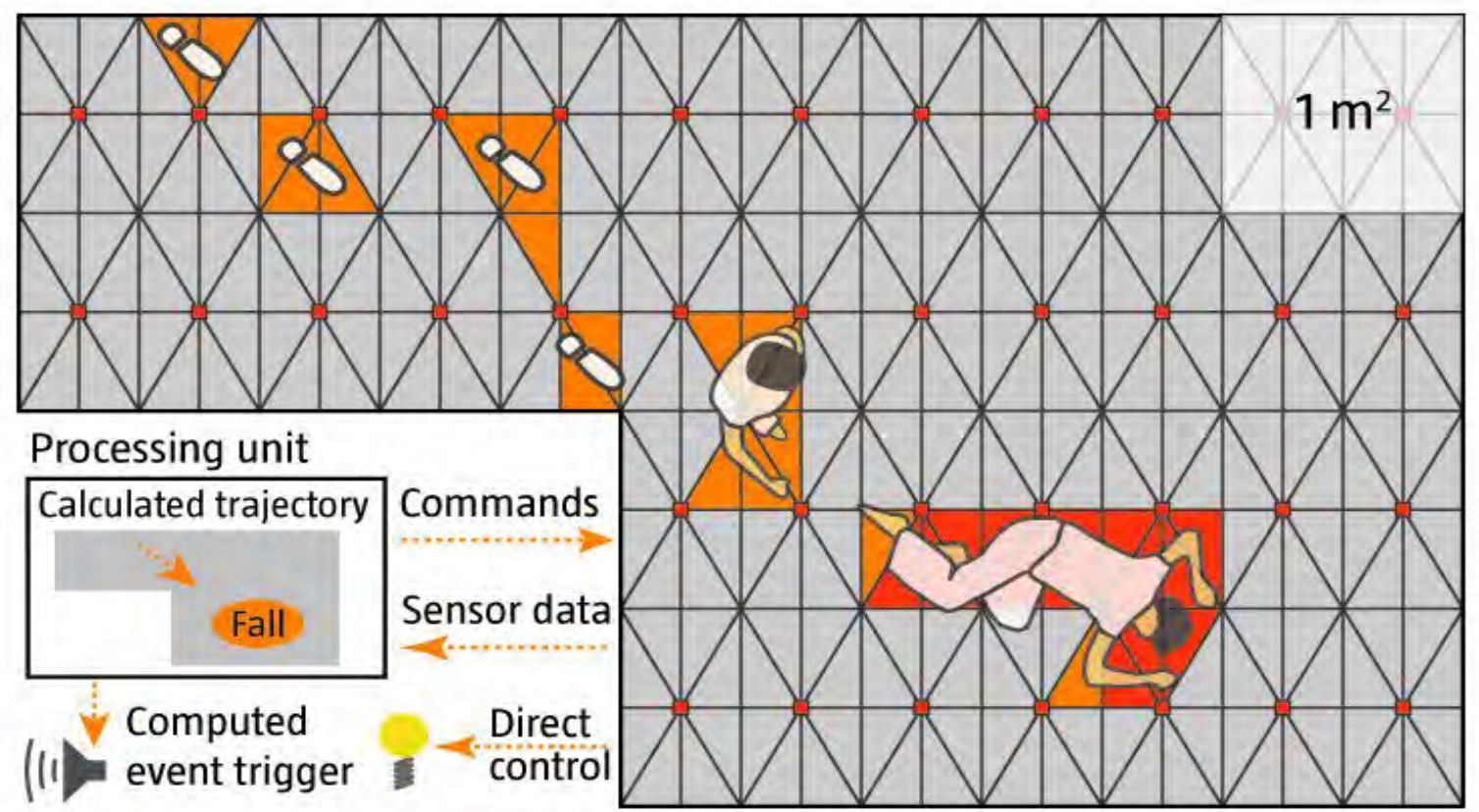

Fig. 1. Schematic of the SensFloor ${ }^{\circledR}$ system. A person walking across the floor generates sensor events on the triangular conductive sensor fields which are sent out wirelessly by the radio modules. In this example, the spatial resolution is reduced to 16 fields per $\mathrm{m}^{2}$. A fall can be identified based on its characteristical sensor pattern. Receivers in the room either control appliances directly by means of relays or send the data to a processing units (e.g. an embedded PC) which analyses the data to detect events such as a fall. The processing unit can be integrated into the home automation system.

active sensor electronics. The sensing underlay contains a grid of radio modules each of which is connected to eight sensor areas of the conductive fleece. The modules' flexible printed circuit boards are specially adapted to the integration in the textile underlay [4]. A typical grid distance of the sensor modules is $50 \mathrm{~cm}$, which leads to a spatial sensor resolution of 32 sensor fields per square meter. However, the actual geometry of the sensor layout is generated by a cutting plotter based on CAD data and is, therefore, very flexible. Furthermore, as the base material is textile, the underlay can be cut at the installation site to adapt to any given room geometry.

The sensor principle is based on frequently measuring the capacitance of each sensor field through charge/discharge cycles to obtain an averaged capacitance of each field with a typical frequency of about 10 times per second. The averaging is necessary to filter out electromagnetic noise induced by perturbations from appliances nearby.

As known from the working principle of capacitors, the capacitance measured for the sensor fields depends strongly on the distance and material of nearby objects with high permitivity (dielectric constant) such as metal, water or the human body. To account for the permitivity of the floor covering, a baseline capacitance is measured each time the sensor modules are powered up. This way, also the effect of reduced capacitance of smaller sensor fields due to cutting the underlay during installation is compensated.

As the human body has a high dielectric permitivity, footsteps on a floor equipped with SensFloor ${ }^{\circledR}$ lead to measurable changes in capacitance of the sensor fields underneath. However, the variations in capacitance are very small compared to the base line value and often even smaller than the noise level. Therefore, the quality of the measurement- and filter algorithms must be very high.

A sensor event is defined when the measured increase in capacitance of one or more sensor fields belonging to an electronics module exceeds a configurable level.

Once a sensor event is detected, it is sent out wirelessly by means of a radio transceiver on each sensor module which works on the $868 \mathrm{MHz}$ ISM band. The event messages have a proprietary format and contain an individual address which identifies the module and thus, the location within the room where the sensor event was generated. In addition, the 


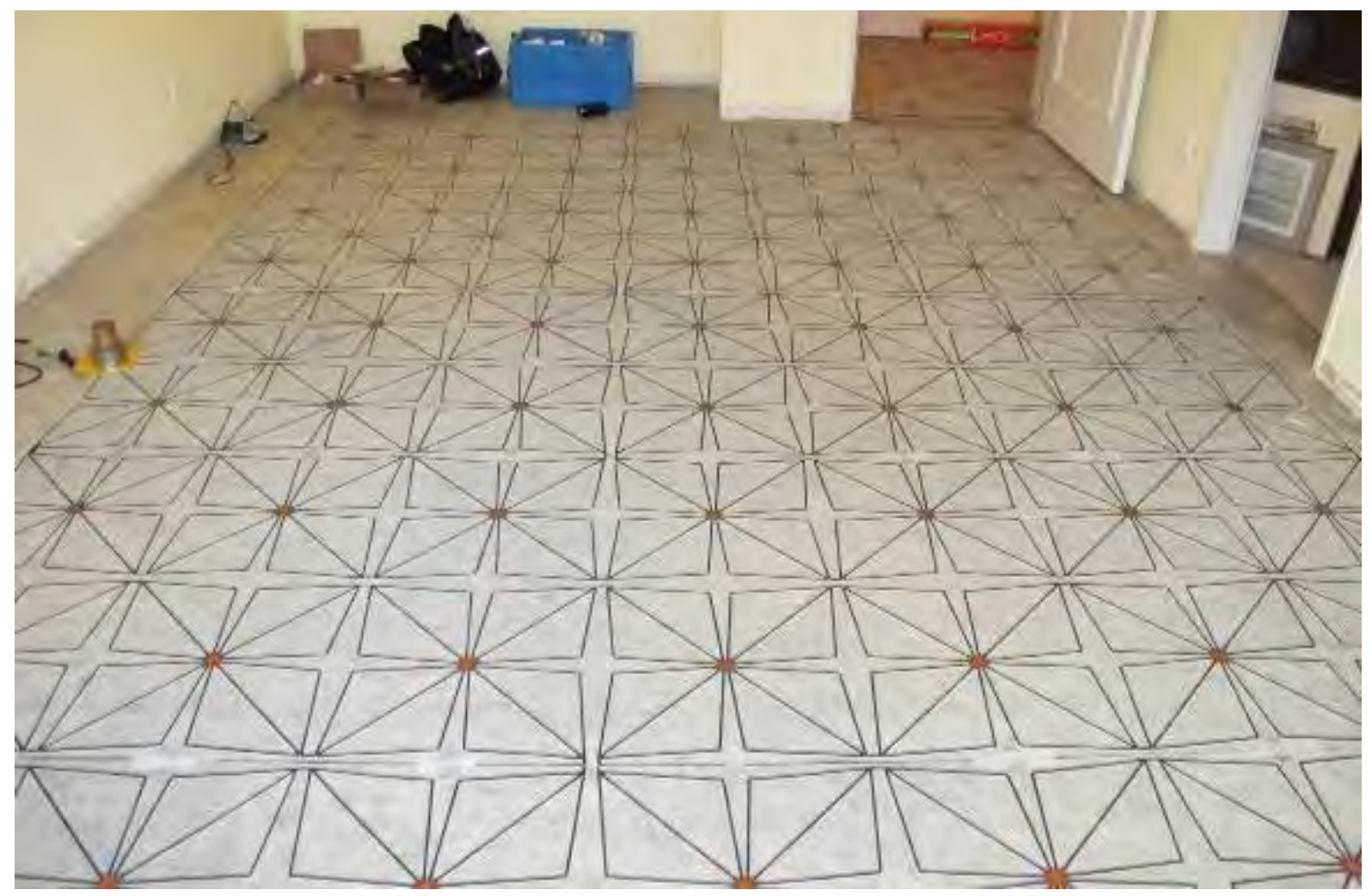

Fig. 2. Sensor underlay installation under progress at the assisted living apartment from Fig. 4 (Living room, corridor). The underlay has 4 radio modules and 32 sensor areas per $m^{2}$

capacitance of the eight sensor fields at the moment of the sensor event is transmitted.

The wireless data packages are received by one ore more transceivers in the environment which are programmed to react on sensor events depending on the defined application.

Applications range from simply switching an orientation light when any part of the floor is activated, e.g. when the person steps out of bed, to more complex logical or pattern analysis of the sensor data. In the latter case, the transceiver processes the sensor events, analyses the time series and reconstructs the movement trajectories of the people walking on the floor. Based on this information, the transceiver is able to control wireless switches which can operate e.g. automatic doors, alarm devices, lights, heating, and traffic counters. It is also possible to send commands to an already existing building control network. Transceivers with a MIDI or a PS/2 interface are also available.

As the data coming from the floor sensors is always the same, independent of the specific application realized by the receivers and the "meaning" of the data is defined only by the address (i.e. the location) of the modules, no specific pairing between sender and receiver is necessary and the communication overhead is low. As there is usually no communication between the sensor modules, it is in principle possible that radio packets are lost due to collision in the transmission channel. However, all applications of the sensor floor are based on evaluating whole populations of sensor events rather than single signals. Therefore, missing some events does not impair the overall function of the system. If required, bidirectional data transmission can be used to request the status of the modules (self-test), to reconfigure their transmission characteristics or to acquire the temperature of the modules, which is determined by a built-in temperature sensor on each module.

\section{Concrete Implementation}

Figure 2 shows the installation in an assisted living apartment in Bremen, Germany. The underlay was produced in a width of 1 meter and a length of 50 meters. Multiple lanes were installed side by side with a mechanical and electrical connection made by conductive adhesive tape. The underlay needs a supply voltage of 9 to 12 Volts which can be delivered by a single AC/DC power adapter with functional ground (Fig. 2 on the left). 


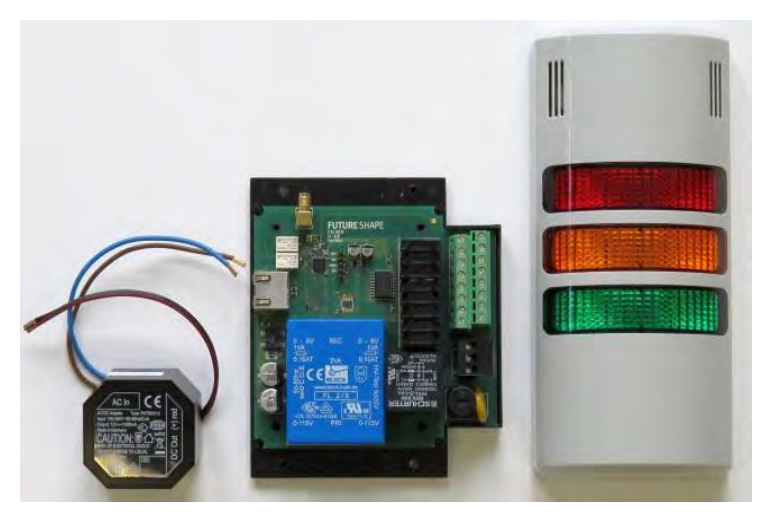

Fig. 3. The built-in power supply for the sensor underlay (left) and the transceiver (centre) for the control of $A A L$ applications. It features 8 relays for $230 \mathrm{~V}$ devices, 2 potential-free relays, as well as a serial interface. The signal tower (right) outside of the room indicates activity, fall or deactivation of the system.

For the connection of the transceiver to home automation or indoor call systems a serial interface and two potential-free relays are implemented, as well as eight relays for controlling 230 Volts devices (Fig. 3, center). The max. power dissipation per sensor module is $25 \mathrm{~mA}$ at $9 \mathrm{~V}$.

A helpful accessory for nursing homes is a signal tower outside of the room indicating activity, fall or deactivation of the system (Fig. 3 right).

Figure 4 shows the ground plan of the installation in Fig. 2 and the functions supported by the SensFloor ${ }^{\circledR}$ :

1. Access control at the entrance door. The SensFloor can be combined with RFID readers or other identification systems.

2. Activity monitoring: for instance, if a person stays in the bathroom longer than usual, an accident may have happened.

3. Switching on an orientation light as soon as someone is in contact with the floor.

4. The SensFloor underlay can also be installed underneath the mattress as a bed sensor for detecting sleep movements.

5. Fall detection, using pattern recognition.

6. The control of automatic doors can be improved: the door opens only if a person directly walks towards it and saves unnecessary opening cycles for persons walking in parallel to the door. The SensFloor can also detect a wheelchair.

7. Switch-off dangerous appliances at leave.

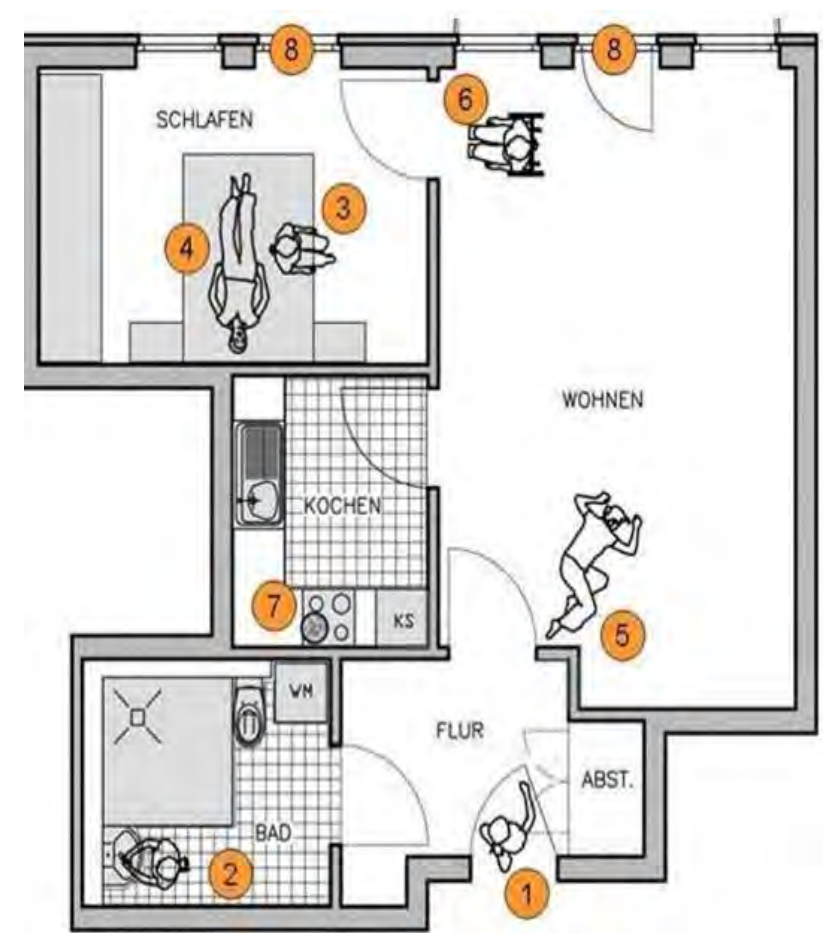

Fig. 4. Applications of the sensor floor installation within an assisted living apartment: (1) access control, (2) activity monitoring, (3) orientation light, (4) bed sensor, (5) fall detection, (6) control of automatic doors, (7) switch-off at leave, (8) intrusion alarm

\section{Intrusion alarm at the windows.}

Energy saving applications by controlling light, heating and air condition based on the number of people present is also possible.

\section{Summary}

We have presented an innovative sensor system which can be embedded invisibly into the floor and which ambiently assists people in AAL application scenarios.

Concrete implementations in the domain of health care have already been realized. However, implementations are also possible in any other domain where the knowledge of the location, number and state of people is of advantage.

The development of the SensFloor system was supported by the German Ministry for Education and Research (BMBF) under the project number FKZ 16SV3936.

\section{References}

[1] Steinhage, A., Lauterbach, C., Monitoring Movement Behaviour by Means of a Large-Area Proximity Sensor Array in the Floor, BMI 2008: pp. 15-27. 
[2] Haritaoglu, I., Harwood, D., Davis, L., W4: Real-Time Surveillance of People and Their Activities, IEEE Trans. on Pattern Recognition and Machine Intelligence, Vol. 22, No. 8, 2000.

[3] Richardson, R., Paradiso, J., Leydon, K., Fernstrom, M., Z-tiles: Building blocks for modular pressure-sensing, Proc of Conf. on Human
Factors in Computing Systems Chi04, 2004, pp. 1529-1532

[4] C. Lauterbach, A. Steinhage, A. Techmer, M.M. Jakob, C.S. Nowakowski, W. Pessenhofer: "Large-area smart textiles", in: Y. Sun, G. Chen, S. S. Wang: World Journal of Engineering 7 (2) 2010, pp. 266-271. 